\title{
Inverse transport for the verification of the Comprehensive Nuclear Test Ban Treaty
}

\author{
J.-P. Issartel ${ }^{1, *}$ and J. Baverel ${ }^{1}$ \\ ${ }^{1}$ Ecole Nationale des Ponts et Chaussées, Centre d'Enseignement et de Recherche en Environnement Atmosphérique, France \\ *Previously at Commissariat à l'Energie Atomique, DASE, Bruyères le Châtel, France
}

Received: 1 August 2002 - Published in Atmos. Chem. Phys. Discuss.: 19 November 2002

Revised: 7 April 2003 - Accepted: 22 April 2003 - Published: 19 May 2003

\begin{abstract}
An international monitoring system is being built as a verification tool for the Comprehensive Test Ban Treaty. Forty stations will measure on a worldwide daily basis the concentration of radioactive noble gases. The paper introduces, by handling preliminary real data, a new approach of backtracking for the identification of sources of passive tracers after positive measurements. When several measurements are available the ambiguity about possible sources is reduced significantly. The approach is validated against ETEX data. A distinction is made between adjoint and inverse transport shown to be, indeed, different though equivalent ideas. As an interesting side result it is shown that, in the passive tracer dispersion equation, the diffusion stemming from a time symmetric turbulence is necessarily a selfadjoint operator, a result easily verified for the usual gradient closure, but more general.
\end{abstract}

\section{Introduction}

We describe a new method for locating the source of a tracer after atmospheric concentration measurements. It applies to passive tracers or to tracers subject to some linear decay processes such as radioactive decay or rain scavenging. The connection of backtracking with the adjoint transport equation has been mentioned long ago (Uliasz and Pielke, 1991; Pudykiewicz, 1998). An extensive theory of adjoint equations has been proposed by Marchuk (1992). In Sects. 2 and 3 we state, with the theoretical consequences, our point of view that the two approaches of backtracking, inverse transport and adjoint transport, are firstly different and secondly equivalent. This will enable to take each measurement into account through a computationally cost effective retroplume representing the air of the sample scattered back in time according to a dispersion equation both adjoint or inverse. Sev-

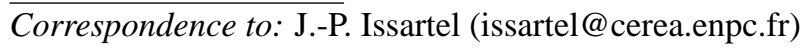

eral retroplumes may be combined together in order to reveal in a non statistical quantitative manner which sources are compatible with the corresponding measurements.

Our questions originally arose from the Comprehensive Test Ban Treaty (De Geer, 1996; Carrigan et al., 1996). An international system of forty stations will provide a worldwide monitoring of radioactive gases produced by the tests. ${ }^{133} \mathrm{Xe}$, the main one, may be released as well by nuclear plants (Kunz, 1989) so that ambient concentrations may interfere with the detection of nuclear tests. After underwater tests ${ }^{133} \mathrm{Xe}$ reaches quickly the atmosphere. It exhales through faults during tenths of hours after underground tests. An atmospheric test would jointly release radioactive aerosols. Such aerosols as ${ }^{140} \mathrm{Ba}$ are accurately monitored, in the frame of the Treaty, by an eighty station network. Nevertheless they are removed from the atmosphere by the rains, and above all they are not released after underground or underwater explosions. When ${ }^{133} \mathrm{Xe}$ is detected in the absence of aerosols the source to be identified is probably a fixed point at ground or sea level from where the gas possibly spread during hours. Only such sources will be considered hereafter. They are met in a lot of industrial circumstances (Goyal and Singh, 1990; Sharan et al., 1995; Baklanov, 2000; Gallardo et al., 2002; Olivares et al., 2002).

The method will be presented with data produced by the CTBT station of Freiburg im Breisgau, Germany, where several types of detectors were calibrated during 2000. A $100 \mathrm{mBq} \cdot \mathrm{m}^{-3}$ peak of ${ }^{133} \mathrm{Xe}$, ten times above the background, was detected on 3 February 2000, in a sample taken by a U.S. detector between 2:00 and 10:00 UT (Arthur et al., 2001). It has not been possible to confirm any source for this event. This lack enables to freely explore general principles without paying too much attention to the limited quality of the input meteorological fields or parameterisations. This necessary effort is finally addressed in the frame of the ETEX experiment sponsored in 1994 by the European Commission and described by the Joint Res. Centre (1998) who provides 
the data on its web site (http://java.ei.jrc.it/etex/database/).

The calculations were performed with the atmospheric transport model POLAIR (Sportisse et al., 2002; Sartelet et al., 2002) developed at the Centre d'Enseignement et de Recherche Eau, Ville, Environnement. POLAIR is the fruit of a close cooperation with the team in charge at Electricité de France of the passive atmospheric transport model Diffeul (Wendum, 1998). It is a fully modular three dimensional Eulerian chemistry transport model. Advection is solved by a third order direct space time scheme with a Koren-Sweby flux limiter function (Sweby, 1984; Koren, 1993) as advocated by Spee (1998); diffusion, parameterised according to Louis (1979), is solved by a classical three point scheme. The reactive part of the model was switched off for the present application. In order to cover western Europe we used a grid extending from $15^{\circ} \mathrm{W}$ to $35^{\circ} \mathrm{E}$ and for the Freiburg episode: $35^{\circ} \mathrm{N}$ to $70^{\circ} \mathrm{N}$, for ETEX: $40^{\circ} \mathrm{N}$ to $67^{\circ} \mathrm{N}$. Outer clean air boundary conditions were used. The horizontal resolution of the model was $0.5^{\circ} \times 0.5^{\circ}$ with fourteen levels at 32,150 , $360, \ldots, 6000 \mathrm{~m}$ above ground or sea level and a 15 minute time step. Meteorological data produced by the European Centre for Medium Range Weather Forecast were kindly supplied by Météo France. These six-hourly data had the same horizontal resolution as POLAIR but had to be interpolated according to the time steps and to the vertical Cartesian levels of the model.

For the convenience of the reader we give hereafter the list of the symbols defined and used later in the text with their SI units. "uat" means: "unit amount of tracer".

$\begin{array}{ll}M_{s}, M_{d}, M_{\text {ex }} \mathrm{kg} \\ \varepsilon & \mathrm{kg}^{-1} \\ \rho & \mathrm{kg} \mathrm{m}^{-3} \\ v & \mathrm{~m} \mathrm{~s}^{-1} \\ \lambda & \mathrm{s}^{-1} \\ \kappa & \mathrm{m}^{2} \mathrm{~s}^{-1} \\ \chi & \text { uat kg }^{-1} \\ \sigma & \text { uat kg-1 } \mathrm{s}^{-1} \\ \hat{\chi} & \text { uat kg } \\ \hat{\sigma} & \text { uat kg-2 } \mathrm{s}^{-1} \\ c & \text { uat } \mathrm{m}^{-3} \\ s & \text { uat } \mathrm{m}^{-3} \mathrm{~s}^{-1} \\ \chi^{*} & \text { unitless } \\ \pi & \mathrm{s}^{-1} \\ \hat{\chi} & \mathrm{kg}^{-1} \\ \hat{\pi} & \mathrm{kg}^{-1} \mathrm{~s}^{-1} \\ \mu(\chi, \pi) & \text { uat } \\ \mu(\chi, \hat{\pi}) & \text { uat kg-1} \\ \mu(\hat{\chi}, \hat{\pi}) & \text { uat kg-2 } \\ Q & \text { uat } \\ \end{array}$

Depending on its nature many units may be used to describe an amount of the tracer. Accordingly "uat" may correspond to kilogrammes for a mass, Becquerel for an activity, Coulomb for a charge... In theoretical situations we shall consider the air itself as a tracer with uat $=1 \mathrm{~kg}$; we shall also consider scalar tracers with no unit, then uat $=1$. Note that adjoint tracers are always considered in one or other of these theoretical situations.

\section{Inverse transport}

Before entering the technical details of this section, let's try to give in simple words the main motivations. Backtracking is generally addressed as a sensitivity analysis. The idea that a concentration measurement is influenced by sources leads to using an adjoint transport (Robertson and Persson, 1993; Penenko and Baklanov, 2001). We shall privilege in this paper the other intuitive point of view that the air sampled for the measurement has arrived from somewhere. This will lead to the inverse transport Eq. 5. The two ideas are generally not clearly distinguished: we think they are very different. The sensitivity idea leads to an adjoint transport equation including an adjoint diffusion. The backward idea leads to an inverse transport Eq. 5 including the same diffusion as the forward transport equation. In order to see these ideas are equivalent much attention must be paid, as explained below, to a mathematically appropriate choice of conventions, mainly the physical units of the variables entering the description of the measurement as a scalar product. Unfortunately the important things go through the mathematical quibbling. A result of this equivalence is that diffusion must be a self-adjoint operator. This general result should not be mistaken for the computational obviousness that the Fickian gradient closure of diffusion is self-adjoint.

The idea of inverse transport bears the very intuitive concept of "retroplume". The air sampled for a measurement is made up of particles that have been travelling separately before gathering inside the detector. In order to understand the meaning of a measurement it is desirable to know more about the history of this air. Rebuilding the individual history of each particle is neither possible nor useful. This history must be addressed in macroscopic terms by just examining the concentration of this air among the ambient air before the sample was taken. This concentration is well defined, or at least as well defined as the concentration of a standard plume. The difference is that the "historical concentration" of the sample is homogenised towards the past while the concentration of a standard plume is homogenised towards the future. We shall use the word "retroplume" as a generic term to designate the past history of a sample, or its macroscopic past history, or even more specifically its historical concentration. This definition displays a past-future symmetry all the more embarrassing as it corresponds to the all-day experience: when we are in a room, we do not know where the air of the room will be spread the day after, but we symmetrically do not know where it was spread the day before. This embarrassment, tied to the second principle, will be analysed in a forthcoming paper. 
The technical description of inverse transport required for the applications is not so intuitive. In particular we shall generally use "normalised retroplumes". Let's consider the release, dispersion and measurement of a tracer passively transported by the motion of the air. All information about this passive transport between parts of the atmosphere may be summed up by an exchange ratio. We introduce two volumes $\mathcal{S}$ at time $t_{s}, \mathcal{D}$ at time $t_{d}, t_{s} \leq t_{d}$. In practice these volumes will be in our model the grid meshes of the source of tracer and of the detector. The mass of the air contained in $\mathcal{S}$ at $t_{s}$ and $\mathcal{D}$ at $t_{d}$ are denoted $M_{s}, M_{d}$, the mass of all air particles exchanged by the two volumes in the prescribed delay is denoted $M_{e x}$. The exchange ratio is defined as:

$\varepsilon\left(\mathcal{S}, t_{s}, \mathcal{D}, t_{d}\right)=\frac{M_{e x}}{M_{s} M_{d}}$

This ratio equally describes the dispersion of the air from $\mathcal{S}$ or the origin of the air in $\mathcal{D}$. To see that let's denote $\chi(\boldsymbol{x}, t)$ the local concentration per unit mass of air after $t_{s}$ of the plume of air from $\mathcal{S}$; the total amount of air released as a self tracer is $M_{s}$. We symmetrically denote $\chi^{*}(\boldsymbol{x}, t)$ the concentration before $t_{d}$ of the retroplume of the air sampled in $\mathcal{D}$; the total amount of air now considered an inverse selftracer is $M_{d}$. In this theoretical context with the air as a self forward or backward tracer, the unit amount of tracer is the kilogramme (of air). $\chi$ and $\chi^{*}$ are both unitless mass mixing ratios. The mass exchanged and exchange ratio may be evaluated as:

$$
\begin{aligned}
M_{e x} & =\int_{\mathcal{D}} \rho \chi\left(\boldsymbol{x}, t_{d}\right) d \boldsymbol{x}=\int_{\mathcal{S}} \rho \chi^{*}\left(\boldsymbol{x}, t_{s}\right) d \boldsymbol{x} \\
\varepsilon & =\frac{1}{M_{d}} \int_{\mathcal{D}} \rho \frac{\chi\left(\boldsymbol{x}, t_{d}\right)}{M_{s}} d \boldsymbol{x}=\frac{1}{M_{S}} \int_{\mathcal{S}} \rho \frac{\chi^{*}\left(\boldsymbol{x}, t_{s}\right)}{M_{d}} d \boldsymbol{x}
\end{aligned}
$$

We thus introduce as announced the normalised plume and retroplume with concentrations $\hat{\chi}=\frac{\chi}{M_{s}}, \hat{\chi}^{*}=\frac{\chi^{*}}{M_{d}}$ tied to total forward and backward releases both equal to unity. We obtain (Hourdin and Issartel, 2000) a reciprocity relation; the overbars stand for averages of $\hat{\chi}$ in $\mathcal{D}$ at $t_{d}$ and $\hat{\chi}^{*}$ in $\mathcal{S}$ at $t_{s}$ :

$\varepsilon\left(\mathcal{S}, t_{s}, \mathcal{D}, t_{d}\right)=\overline{\hat{\chi}\left(\mathcal{D}, t_{d}\right)}=\overline{\hat{\chi}^{*}\left(\mathcal{S}, t_{s}\right)}$

An amount $Q$ of tracer released in $\mathcal{S}$ at $t_{S}$ generates a plume with an average concentration per unit mass of air $Q \varepsilon\left(\mathcal{S}, t_{s}, \mathcal{D}, t_{d}\right)$ measured in $\mathcal{D}$ at $t_{d}$. The same amount $Q$ released in $\mathcal{D}$ at $t_{d}$ and transported back in time will lead to the same average concentration per unit mass $Q \varepsilon$ in $\mathcal{S}$ at $t_{s}$. Normal, forward transport and this backtracking are equivalent and accordingly the analytic description of the second will be readily deduced from that of the first. In the case of advection-diffusion with a wind-field $v$ and a diffusion operator $\zeta$, the analytic equation for backward transport is obtained exactly by the same averaging procedure of the individual motions of the particles:

$\frac{\partial \hat{\chi}}{\partial t}+\boldsymbol{v} \cdot \nabla \hat{\chi}+\zeta(\hat{\chi})=\hat{\sigma}$ $-\frac{\partial \hat{\chi}^{*}}{\partial t}-\boldsymbol{v} \cdot \nabla \hat{\chi}^{*}+\zeta\left(\hat{\chi}^{*}\right)=\hat{\pi}$

Note that the theoretical diffusion operator $\zeta$ is considered in these equations independently of the later choice of a closure. The normalised release in $\mathcal{S}$ at $t_{s}$, in $\mathcal{D}$ at $t_{d}$, are described by the normal source function $\hat{\sigma}=\frac{\sigma}{M_{s}}$ and by the normal detector function $\hat{\pi}=\frac{\pi}{M_{d}}$ below; the symbol $\delta_{\text {time }}(t)$ is a Dirac function of the time so that its physical unit is the inverse unit of time:

$\hat{\sigma}(\boldsymbol{x}, t)=\frac{\delta_{\text {time }}\left(t-t_{s}\right)}{M_{S}} \quad$ in $\mathcal{S}, \quad 0$ outside

$\hat{\pi}(\boldsymbol{x}, t)=\frac{\delta_{\text {time }}\left(t-t_{d}\right)}{M_{d}} \quad$ in $\mathcal{D}, \quad 0$ outside

In a more general situation a source function may be largely spread in space and time, for instance the source of carbon dioxide. This is true as well for the detector function. The detector may deliver time averaged measurements corresponding to the time interval when the sample was taken, it may furthermore be airborne with a varying position.

The diffusion operator $\zeta$, unlike the winds, has the same sign in Eqs. 4 and 5: diffusion symmetrically dilutes the fate of $\mathcal{S}$ and the origin of $\mathcal{D}$. This is true provided the microscopic (i.e. in practice sub-grid scale) turbulent motions averaged into a macroscopic diffusion are statistically time symmetric, thus never privileging a direction with respect to the opposite one; the condition is not satisfied by convective turbulence. The obstacle of an unphysical anti-diffusion classically restricting backtracking to the Lagrangian investigation of an individual backtrajectory is avoided in this Eulerian approach. This Eulerian approach is equivalent to the Lagrangian technique of calculating back in time the trajectories of a great number of particles departing from the detector and subject to the same diffusion as in the forward model. The resolution of the Lagrangian calculation of the retroplumes will be nevertheless limited by the number of particles.

\section{Inverse transport versus adjoint transport}

Equation 5 is a macroscopic description of the history of the air sampled by the detector. It is as well a sensitivity equation, i.e. an adjoint equation as we now explain. Notice that the measurement $\mu$ behaves as a scalar product of the tracer concentration and of the detector function:

$\mu(\chi, \hat{\pi})=\int_{\Omega \times \mathrm{T}} \rho(\boldsymbol{x}, t) \chi(\boldsymbol{x}, t) \hat{\pi}(\boldsymbol{x}, t) d \boldsymbol{x} d t$

The integration is over the atmosphere $\Omega$ and the time domain T. $\rho$ is the density of the air. In this equation the concentration of tracer, an amount of tracer per unit amount of air, is no longer normalised but the detector function is still normalised with respect to the mass of the sample so that the 
backward concentration $\hat{\chi}^{*}$ turns out a unitless mass mixing ratio. Hence the measurement $\mu(\chi, \hat{\pi})$ is an amount of tracer per unit mass of air (of the sample). Note that $\mu(\chi, \pi)$ is the amount of tracer in the sample; $\mu(\hat{\chi}, \hat{\pi})$ would be the source detector exchange ratio of Eq. 3 .

Let $\mathcal{L}$ and $\mathcal{L}^{*}$ be the linear operators defined by the forward and backward Eqs. 4 and 5 (or 11 and 12) together with adequate zero boundary conditions: $\chi=\mathcal{L}(\sigma), \hat{\chi}^{*}=\mathcal{L}^{*}(\hat{\pi})$. The measurement $\mu$ tied to any source $\sigma$ and sampling distribution $\hat{\pi}$ decomposes according either to elementary samples, $\delta \mu_{x}=\rho \chi \hat{\pi}(\boldsymbol{x}, t) d \boldsymbol{x} d t, \mu=\int \delta \mu_{x}$, or to elementary releases $\delta \mu_{y}=\rho \sigma \hat{\chi}^{*}(\boldsymbol{y}, u) d \boldsymbol{y} d u, \mu=\int \delta \mu_{y}(\boldsymbol{y}, u$ : position and time considered adjoint variables). Hence we obtain a general form of the reciprocity relation 3 :

$$
\int_{\Omega \times \mathrm{T}} \rho \mathcal{L}(\sigma) \hat{\pi}(\boldsymbol{x}, t) d \boldsymbol{x} d t=\int_{\Omega \times \mathrm{T}} \rho \sigma \mathcal{L}^{*}(\hat{\pi})(\boldsymbol{y}, u) d \boldsymbol{y} d u
$$

The relation shows how source and detector change roles. As announced the operators $\mathcal{L}$ and $\mathcal{L}^{*}$ are adjoint for the measurement product and so are equations 4 and 5 .

In reactor and neutron transport theory $\hat{\chi}^{*}(\boldsymbol{x}, t)$ is called the "importance" for the measurement of a particle released in $\boldsymbol{x}$ at $t$ (Lewins, 1965).

The adjoint interpretation of inverse transport implies that, for appropriate conventions, the diffusion tied to a timesymmetric turbulence is self-adjoint:

$\zeta=\zeta^{*}$ i.e. $\int \rho \chi \zeta\left(\chi^{*}\right) d \boldsymbol{x} d t=\int \rho \zeta(\chi) \chi^{*} d \boldsymbol{y} d u(10)$

The self-adjoint constraint is fulfilled by the classical Fickian gradient diffusion used in POLAIR with a coefficient $\kappa$ : $\zeta(\chi)=-\frac{1}{\rho} \nabla \rho \kappa \nabla \chi$. Once the measurement product has been put into the appropriate form 8 this is a computational obviousness. The result 10 is more general anyway as it concerns diffusion itself before, as already stressed, the choice of a closure. Accordingly only self-adjoint operators should be proposed as a relevant closure of diffusion. This general property may be compared to the similar property of the linearised diffusive collision operator of the Boltzmann transport equation for particles in the position-velocity space of kinetic theory (McCourt et al., 1990).

During its transport by the motions of the air ${ }^{133} \mathrm{Xe}$ undergoes a linear decay. Its half life $\tau_{1 / 2}=5.5$ days corresponds to a constant $\lambda=\log 2 / \tau_{1 / 2}$. It is possible to take this decay into account for the backward calculations. Just like diffusion it has exactly the same effect in the inverse world as in the direct world: $\chi^{*}$ decays towards the past the same way as $\chi$ decays towards the future. This may be surprising but merely means that, because of the losses of tracer, the importance of ancient sources for the measurement is attenuated. Forward and backward equations associated to ${ }^{133} \mathrm{Xe}$ with a vertical gradient diffusion read as:

$$
\frac{\partial \chi}{\partial t}+\boldsymbol{v} \cdot \nabla \chi-\frac{1}{\rho} \frac{\partial}{\partial z}\left(\rho \kappa \frac{\partial \chi}{\partial z}\right)+\lambda \chi=\sigma
$$

$$
-\frac{\partial \hat{\chi}^{*}}{\partial t}-\boldsymbol{v} \cdot \nabla \hat{\chi}^{*}-\frac{1}{\rho} \frac{\partial}{\partial z}\left(\rho \kappa \frac{\partial \hat{\chi}^{*}}{\partial z}\right)+\lambda \hat{\chi}^{*}=\hat{\pi}
$$

We shall stress finally by means of these equations that the analytic form of the measurement product is mainly conventional. It would become $\mu=\int_{\Omega \times \mathrm{T}} c \hat{\pi} d \boldsymbol{x} d t$ with a concentration of tracer $c=\rho \chi$ and a source $s=\rho \sigma$ referred to the unit volume of air (Pudykiewicz, 1998; Elbern and Schmidt, 1999). But then, with $\hat{\pi}$ a (normalised) sampling rate still referred to the unit mass of ambient air, so would be the adjoint concentration $\hat{\chi}^{*}$ unlike $c$. The transport Eq. 13 would have a different appearance from its adjoint Eq. 12. The symmetry of normal and adjoint advection-diffusion would be hidden and so would be the interpretation of the adjoint advectiondiffusion as an inverse advection-diffusion.

$$
\frac{\partial c}{\partial t}+\nabla c \boldsymbol{v}-\frac{\partial}{\partial z}\left(\rho \kappa \frac{\partial}{\partial z} \frac{c}{\rho}\right)+\lambda c=s(\boldsymbol{x}, t)
$$

Note also that the self-adjoint nature of diffusion has been deduced from physical intuition by Uliasz and Pielke (1991) but the generality of their conclusion was limited by the aforementioned choice of conventions and scalar product. An operator $\nabla \kappa \nabla c$ in Eq. 13 or equivalently $\frac{1}{\rho} \nabla \kappa \nabla \rho \chi$ in Eq. 11 might be a suitable self-adjoint closure of time symmetric turbulent diffusion only if the density $\rho$ of the air was constant.

\section{A single measurement}

When on 3 February 2000, a peak of $100 \mathrm{mBq} \cdot \mathrm{m}^{-3}$ was detected, the question of its origin immediately arose. Such a question is generally answered in terms of Lagrangian backtrajectories: the wind field $\boldsymbol{v}(\boldsymbol{x}, t)$ is integrated backward departing from the detector at a time related to the detection. A curve is obtained supposedly passing by the real source.

In order to account for the duration of the measurement, eight hours in Freiburg, or for the random effect of diffusion, the previous calculation would be repeated many times. This amounts to calculating back in time the trajectories of many Lagrangian particles. It is often considered that, if many backtrajectories go back to a certain region, then the source is probably there.

In fact calculating many backtrajectories amounts to calculating the concentration $\chi^{*}(\boldsymbol{x}, t)$ of a retroplume emitted back in time by the detector. If we investigate the origin of a single particle sampled by the detector, the probability density (per unit mass of air) of its past position is $\hat{\chi}^{*}$ calculated for a normalised detector function (the total amount of adjoint tracer released is normalised to the unity). Nevertheless when macroscopic sources are investigated this statistical interpretation of the retroplume is erroneous. If many backtrajectories go back to a certain region this just means that the region contributed much air to the sample and a source there should not be very big to account for the measurement. 
00 UT 3 February 2000

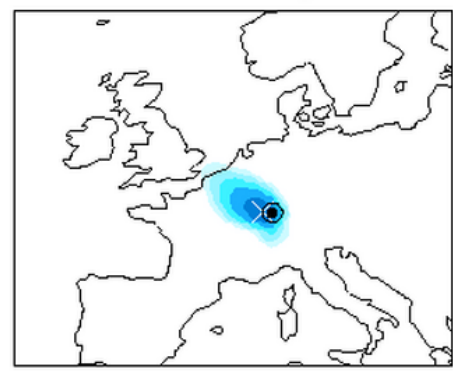

00 UT 1 February 2000

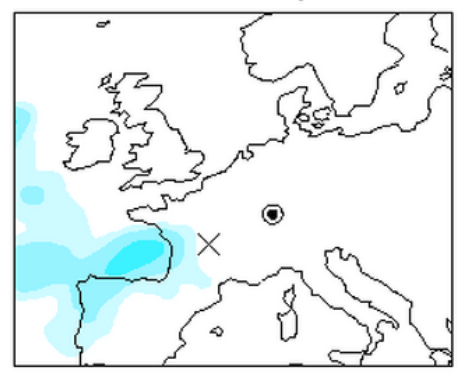

12 UT 2 February 2000

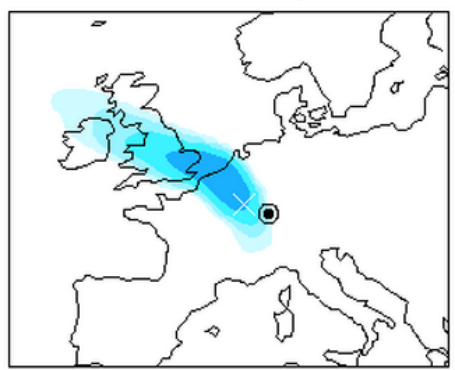

00 UT 31 January 2000

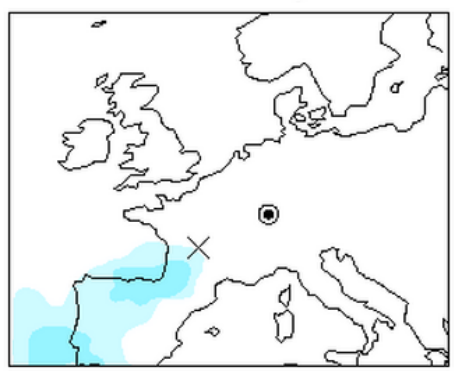

00 UT 2 February 2000

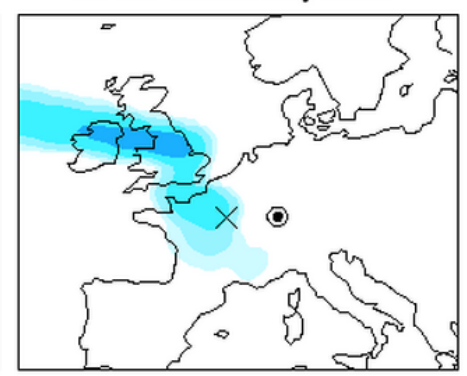

00 UT 30 January 2000

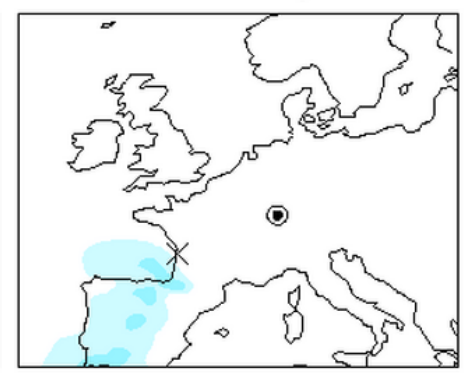

$\mathrm{TBq}$

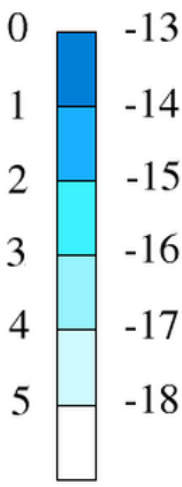

$m^{-3}$

$\log _{10}$

Fig. 1. Normalised retroplume $\hat{\chi}_{1}^{*}(\boldsymbol{x}, t)$ at ground or sea level corresponding to the $100 \mathrm{mBq} \cdot \mathrm{m}^{-3}$ peak in Freiburg between $02: 00$ and 10:00 UT on 3 February 2000. In theory $\hat{\chi}_{1}^{*}$ should be given per kg of air but at ground level it is roughly equivalent and more convenient to give it per $\mathrm{m}^{3}$. The same figure may be read in terms of possible point sources $q=100 \mathrm{mBq} \cdot \mathrm{m}^{-3} / \hat{\chi}_{1}^{*}(\boldsymbol{x}, t)$ with a scale in TBq of ${ }^{133} \mathrm{Xe}$. The circle on the images indicates the position of the detector. The cross describes the backtrajectory of a Lagrangian particle departing back in time from Freiburg on 3 February 2000 at 06:00 UT. Notice that the Lagrangian particle does not follow the centre of the Eulerian retroplume.

A source of intensity $Q$ in $x$ at $t$ will generate a measurement $\mu=\hat{\chi}^{*}(\boldsymbol{x}, t) Q$. In other words a measurement $\mu$ can be explained by an instantaneous point source in $\boldsymbol{x}$ at $t$ of intensity $Q=\frac{\mu}{\hat{\chi}^{*}(\boldsymbol{x}, t)}$. The retroplume establishes a constraint between the position of the source and its intensity.

This deterministic character of the quest for macroscopic point sources was simultaneously clarified, during the technical discussions of the Treaty, by Seibert (2000), describing the retroplume as a source-receptor matrix, and Issartel et al. (2000), a conclusion later adopted in the collective report of an ad-hoc expert group (CTBTO/PTS, 2001).

This analysis easily extends to the case of point sources that are not instantaneous. Suppose a source in $\boldsymbol{x}$ has a rate of release $\dot{Q}(t) \geq 0$ per unit time and a total release is $Q=$ $\int \dot{Q}(t) d t$; the measurement is $\mu=\int \hat{\chi}^{*}(x, t) \dot{Q}(t) d t$. As $\dot{Q}$ is a non-negative function $\mu \leq \max _{t} \hat{\chi}^{*}(\boldsymbol{x}, t) \int \dot{Q}(t) d t$, or:

$Q \geq \frac{\mu}{\max _{t} \hat{\chi}^{*}(\boldsymbol{x}, t)}=Q^{\min }(\boldsymbol{x})$

We still do not know where the source actually lies. It could lie in any position $\boldsymbol{x}$ provided the retroplume went there at some moment. Nevertheless the threshold function $Q^{\text {min }}$ shows that not all positions are equivalent. A source far away from the detector should be greater than a close one.
The retroplume of the peak measurement of ${ }^{133} \mathrm{Xe}$ in Freiburg has been calculated by model POLAIR according to Eq. 12 with a normalised detector function $\hat{\pi}$ concentrated in Freiburg at position $\boldsymbol{x}_{F}$, and during an eight hour interval $\Delta t$; the symbol $\delta_{s p}(\boldsymbol{x})$ is a Dirac function of the space so that its physical unit is the inverse unit of volume:

$\hat{\pi}(\boldsymbol{x}, t)=\left\{\begin{array}{cl}\frac{\delta_{s p}\left(\boldsymbol{x}-\boldsymbol{x}_{F}\right)}{\Delta t \rho\left(\boldsymbol{x}_{F}, t\right)} & 2 \leq t \leq 10 \mathrm{UT}, 2000 / 02 / 03 \\ 0 & \text { otherwise }\end{array}\right.$

Considering Fig. 1 we see that on 2 February 2000, the retroplume moves to the northwest. During the morning of that day it turns to the southwest mainly above the Atlantic. As can be seen on Fig. 2a, an industrial source in Gascony should be as big as $Q^{\mathrm{min}}=1000 \mathrm{TBq}$, orders of magnitude above the exceptional releases by nuclear civilian installations. It seems more reasonable to investigate industrial sources northwest of Freiburg. So far, no such source has been confirmed. The hypothesis has been proposed that the $100 \mathrm{mBq} \cdot \mathrm{m}^{-3}$ peak was related to a very weak medical source in Freiburg. German hospitals use ${ }^{133} \mathrm{Xe}$ for pulmonary investigations. A nuclear test generates $1000 \mathrm{TBq}$ of ${ }^{133} \mathrm{Xe}$ per kiloton, only $10 \%$ exhale in the case of an underground explosion. Regardless of other considerations the 

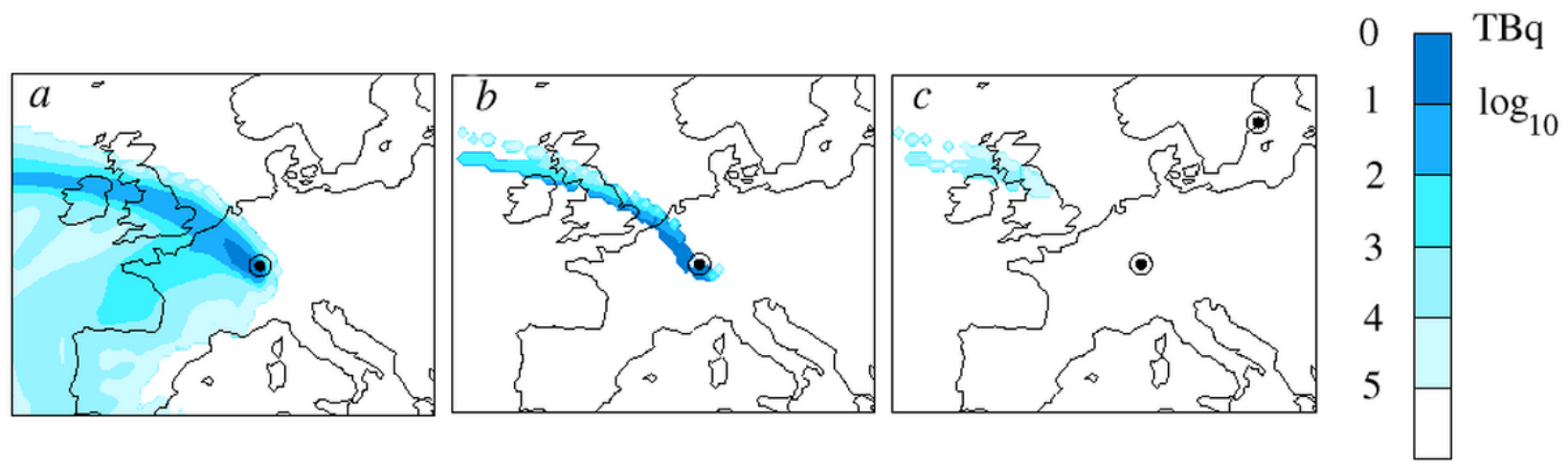

Fig. 2. Minimum release from a point source a ground or sea level (a) $Q^{\min }(\boldsymbol{x})$ compatible with the peak measurement of Freiburg $\mu_{1}=103 \mathrm{mBq} \cdot \mathrm{m}^{-3}$ (b) $Q_{4}^{\min }(\boldsymbol{x})$ compatible with the series of measurements in Freiburg $\mu_{0}=6, \mu_{1}=103, \mu_{2}=42, \mu_{3}=6 \mathrm{mBq} \cdot \mathrm{m}^{-3}$; this value of $Q_{4}^{\min }(\boldsymbol{x})$ would not be altered by considering a fictitious measurement in Stockholm $\mu_{S}=0 \mathrm{mBq}^{-3}$ on 3 February 2000 (c) $Q_{5}^{\min }(\boldsymbol{x})$ compatible with the previous series completed with a fictitious measurement $\mu_{S}=100 \mathrm{mBq} \cdot \mathrm{m}^{-3}$ in Stockholm.

$Q^{\text {min }}$ calculated for most western Europe would be compatible with a 10 kiloton test.

We have also reproduced the traditional diagnostics of investigating a point source in the neighbourhood of an average current line obtained by integrating back in time from the detector the wind field at or close to the ground level. The curve obtained is generally called a "backtrajectory" or even a "Lagrangian backtrajectory". We think the method is ill defined for two reasons. Firstly the real backtrajectory of a real particle is a 3D thing that cannot be obtained by just considering the horizontal wind at some given altitude. Secondly, the wind generally dramatically decreases close to the ground so that the result does depend on the altitude chosen for the calculations. As POLAIR is fundamentally a Eulerian model, we adapted this calculation by just setting vertical advection, horizontal and vertical diffusions to zero. The tracer was advected back in time by the winds obtained at $32 \mathrm{~m}$ above ground or sea level. Because of numerical diffusion (Ouahsine and Smaoui, 1999) a little cloud is formed; this effect remains negligible, the horizontal dimension of the cloud after four days (30 January 2000) are about $200 \mathrm{~km}$, four times less than the advection, ten times less than the extension of the retroplume. Hence we reported on Fig. 1 a cross representing the centre of the little cloud as a realisation of the traditional diagnostics. This backtrajectory first follows the retroplume but too slowly with the ground winds; on 30 January 2000 the cross is at the edge of the retroplume, thousand kilometres away from its centre. It indicates a possible source in Gascony which is very unlikely as previously commented.

\section{Several measurements by a single station}

The diagnosis above can be improved so as to determine minimum total releases $Q_{4}^{\min }(\boldsymbol{x})$ taking into account the con- straints imposed by four measurements obtained in Freiburg. The constraining nature of these additional observations is easy to understand: if the source lies far away, the plume of ${ }^{133} \mathrm{Xe}$ will have much broadened before reaching Freiburg and it will be detected during a long time. If successive measurements in Freiburg display a narrow peak, the source cannot be too far away. In order to evaluate this effect we handled the following four observations (Arthur et al., 2001) with the peak measurement now labelled 1:

$\mu_{0}=6 \mathrm{mBq} \cdot \mathrm{m}^{-3}$ from 18 to $2 \mathrm{UT}, 2000 / 02 / 02-03$

$\mu_{1}=103 \mathrm{mBq} \cdot \mathrm{m}^{-3}$ from 2 to $10 \mathrm{UT}, 2000 / 02 / 03$

$\mu_{2}=42 \mathrm{mBq} \cdot \mathrm{m}^{-3}$ from 10 to $18 \mathrm{UT}, 2000 / 02 / 03$

$\mu_{3}=6 \mathrm{mBq} \cdot \mathrm{m}^{-3}$ from 18 to $2 \mathrm{UT}, 2000 / 02 / 03-04$

As at ground level one $\mathrm{m}^{3}$ roughly contains one $\mathrm{kg}$ of air, we considered the measurements obtained in $\mathrm{mBq} \cdot \mathrm{m}^{-3}$ to be equivalent to measurements in $\mathrm{mBq} \cdot \mathrm{kg}^{-1}$. To each measurement a normal retroplume $\hat{\chi}_{i}^{*}(\boldsymbol{x}, t)$ may be related. A source in $\boldsymbol{x}$ with a rate of release $\dot{Q}(t) \geq 0$ is now subject to the four constraints:

$\mu_{i}=\int \hat{\chi}_{i}^{*}(\boldsymbol{x}, t) \dot{Q}(t) d t \quad i=0,1,2,3$

We considered the following system of constraints were possible errors are taken into account with wide margins (this will be commented in more detail at the end of the section):

$$
\begin{aligned}
& \dot{Q}(t) \geq 0 \\
& 0 \mathrm{mBq} \cdot \mathrm{m}^{-3} \leq \int \hat{\chi}_{0}^{*}(\boldsymbol{x}, t) \dot{Q}(t) d t \leq 10 \mathrm{mBq} \cdot \mathrm{m}^{-3} \\
& 52 \mathrm{mBq} \cdot \mathrm{m}^{-3} \leq \int \hat{\chi}_{1}^{*}(\boldsymbol{x}, t) \dot{Q}(t) d t \leq 206 \mathrm{mBq} \cdot \mathrm{m}^{-3} \\
& 21 \mathrm{mBq} \cdot \mathrm{m}^{-3} \leq \int \hat{\chi}_{2}^{*}(\boldsymbol{x}, t) \dot{Q}(t) d t \leq 84 \mathrm{mBq} \cdot \mathrm{m}^{-3} \\
& 0 \mathrm{mBq} \cdot \mathrm{m}^{-3} \leq \int \hat{\chi}_{3}^{*}(\boldsymbol{x}, t) \dot{Q}(t) d t \leq 10 \mathrm{mBq} \cdot \mathrm{m}^{-3}
\end{aligned}
$$

We want to determine the minimum value $Q_{4}^{\min }(\boldsymbol{x})$ of the total release $\int \dot{Q}(t) d t$ among all admissible rate functions 
$\dot{Q}(t)$. This linear optimisation problem can be easily managed, when time is discretised, by means of the so called "simplex" algorithm. This classical algorithm was first described by Dantzig (1963). We operated it locally, with a one hour time step, for each position $\boldsymbol{x}$ at ground or sea level in western Europe and for sources starting from January the $25^{\text {th }}$. A well known property of the simplex algorithm is that the optimal rate of release $\dot{Q}^{\min }(\boldsymbol{x}, t)$ at position $\boldsymbol{x}$ is non zero for a number of time steps that is at most the number of constraints. The results are reported on Fig. 2b. For most positions the above constraints are not compatible. This means that the ${ }^{133} \mathrm{Xe}$ detected in Freiburg cannot have originated there. The threshold function $Q_{4}^{\text {min }}(\boldsymbol{x})$ is clearly more restrictive than $Q^{\text {min }}$ and the new diagnosis clarifies the previous one. Admissible sources now lie in a narrow strip departing from Freiburg to the northwest through France, Belgium, Great Britain and terminating one thousand kilometres off Ireland. Industrial sources should not be sought further than Wales. The diagnosis clearly excludes the southern part of France where the previous one already allowed only prohibitively big sources. A real advantage is obtained in the western part of France and southern part of England where sources as large as some tenths of kilotons, previously admissible, are now excluded.

Notice that a weak source close to Freiburg is not excluded. The four measurements obtained there might have been contaminated by four little releases from a position nearby. And generally when all the measurements will be from a single station, the same number of local contaminations will make up an admissible source. Therefore, a single station will never be in a position to exclude a source in its close environment. This difficulty can be partly removed if we assume a limited duration of the release. The duration of industrial releases is classically less than twelve hours, one working day. Such a signal, emitted in the neighbourhood of Freiburg, should not interest more than three eight hour samples. It is nevertheless more convenient to use the local optimisation method with information from several stations.

It may be argued that the use of margins in the system of constraints 17 is not compatible with the statistical nature of the measurement errors. In fact the bounds of the margins is determined by our weaker or higher tolerance that the real values of the measurements escape from between them. If we want to be sure that, with $p=99 \%$ probability, the real measurement lie inside the margins, then we have to use wider margins than would be required for $p=90 \%$. Hence the threshold function depends on the prescribed probability: $Q_{4}^{\min }=Q_{4}^{\min }(p, \boldsymbol{x})$.

\section{Measurements from several stations}

The local optimisation method described above is just an abridged way to handle the information contained in the retroplumes. A more accurate understanding of the meteorological situation may require a complete analysis as we now explain. The event detected on 3 February 2000 was observed only in Freiburg. The CTBT station of Stockholm was not yet operating. Let's just imagine what could have been said if a 24 hour sample had been taken there on 3 February 2000. We denote the corresponding normal retroplume as $\hat{\chi}_{S}^{*}$ and the fictitious measurement as $\mu_{S}$. A source in $\boldsymbol{x}$ with a rate of release $\dot{Q}(t)$ would thus lead to $\mu_{S}=\int \hat{\chi}_{S}^{*}(\boldsymbol{x}, t) \dot{Q}(t) d t$.

We imagined two situations. Firstly the requirement that $\mu_{S}=0 \mathrm{mBq} \cdot \mathrm{m}^{-3}$ has been combined with real data from Freiburg in the local optimisation. This additional information would not change the final result. This means that sources previously diagnosed would be mostly compatible with a zero valued measurement in Stockholm in such a way that Fig. $2 b$ is unaltered. Secondly the requirement that $\mu_{S}=100 \mathrm{mBq} \cdot \mathrm{m}^{-3}$ would clearly exclude weak sources close to Freiburg as shown by Fig. 2c. Only big sources northwest of England would be acceptable then.

We now place the transparent figure representing the retroplume $\hat{\chi}_{S}^{*}$ from Stockholm on top of the figure representing the peak retroplume $\hat{\chi}_{1}^{*}$ from Freiburg. Considering the resulting Fig. 3 we appreciate the connections of each point in space and time with both measurements. We first notice that the retroplumes intersect marginally. This is the reason why a zero valued measurement in Stockholm does not alter the local optimisation diagnosis. We still notice that the retroplume from Stockholm does not meet western continental Europe. A source there could not contaminate the sample of Stockholm and would be excluded by the local optimisation diagnosis for a virtual measurement $\mu_{S}=100 \mathrm{mBq} \cdot \mathrm{m}^{-3}$. In that case we notice furthermore that instantaneous spot sources may be considered only in Scotland where the retroplumes intersect marginally. Other acceptable sources should have a duration greater than twelve hours corresponding to the delay separating the passings of one and other retroplumes over most positions.

\section{Real versus analysed winds: ETEX1}

The present section has been developed as a result of the discussion, it was not presented at EGS 2002 Assembly. Whatever the source of the Freiburg episode may have been and wherever it may have lain the transfer of the tracer towards the detectors has been achieved by the real winds of the real atmosphere. The theory of inverse transport is about the real atmosphere but its practical use necessarily goes through a model with analysed winds, parameterisations and numerical schemes. The forward world and the inverse world, ideally identical, are different in practice. This questions the validity of the above analysis of real measurements through retroplumes produced by POLAIR. We thus investigated the first ETEX release of $340 \mathrm{~kg}$ of permethylcyclohexane (pmch) 
00 UT 3 February 2000

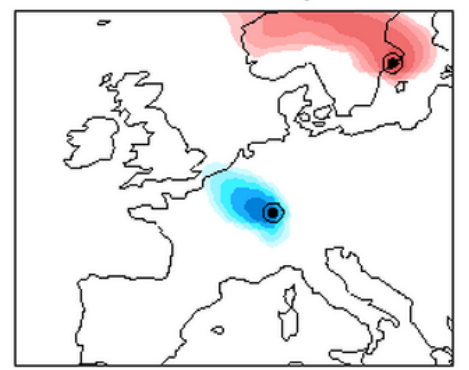

00 UT 1 February 2000

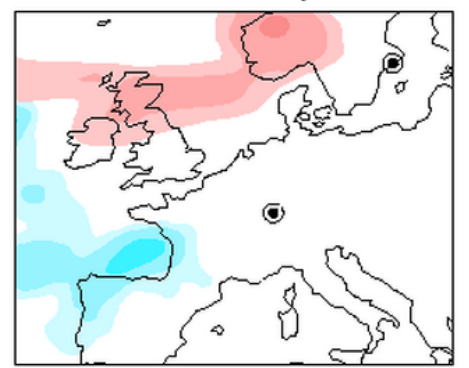

12 UT 2 February 2000

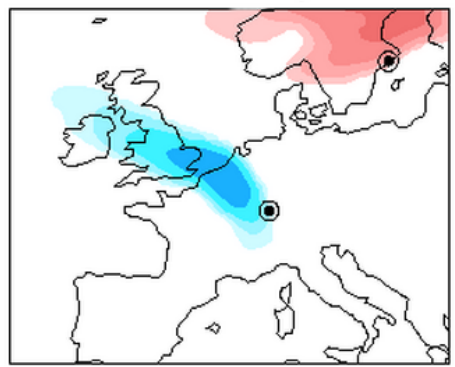

00 UT 31 January 2000

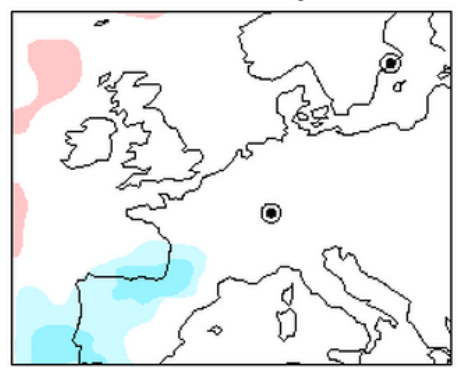

00 UT 2 February 2000

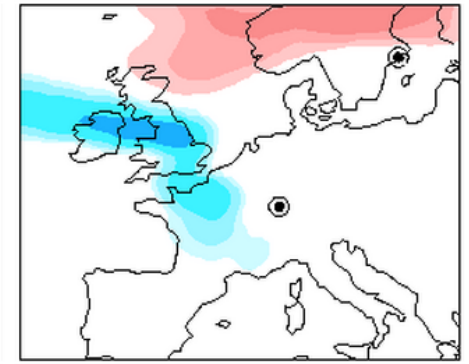

00 UT 30 January 2000

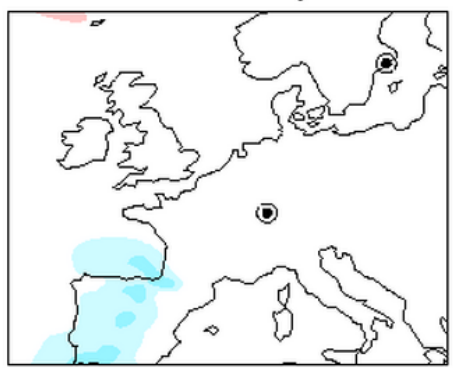

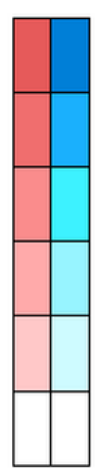

$-13$

$-14$

$-15$

$-16$

$-17$

$-18$

$m^{-3}$

$\log _{10}$

Fig. 3. In blue the normalised retroplume $\hat{\chi}_{1}^{*}$ associated to the peak detection of ${ }^{133} \mathrm{Xe}$ in Freiburg. In red the normalised retroplume emitted by the virtual measurement sampled in Stockholm between 00:00 and 24:00 UT on 3 February 2000. The retroplumes intersect only marginally in Scotland. The measurements are fundamentally independent. Scotland is in fact the only possible position for an instantaneous point source to contaminate positively both measurements. Both retroplumes flow over Ireland or above the ocean West of Ireland but never simultaneously. A point source there could contaminate both measurements but it could not be an instantaneous source.

from Monterfil, Brittany, France, between 23 October 94, 16:00 UT and 24 October 4:00 UT. Time averaged concentration measurements were delivered each third hour by 168 detectors all over Europe. We have selected three stations: F8, Brest, France, west of Monterfil never saw the cloud of pmch and delivered only zero-valued measurements; F20, Reims, France and D10, Essen, Germany were successively on the main way of the cloud. In order to compare the practical achievement of the local optimisation method and the ideal situation of identical forward and backward world we prepared a set of synthetic measurements with POLAIR for a source at the prescribed position and time interval. In the following lines the word "practical" will refer to the real situation (forward real world-backward modelled world) and the word "ideal" to the idealised situation (forward modelled world-backward modelled world). We performed a local optimisation, practically and ideally, with various combinations of F8, F20, D10. The only effect of the zero-valued series of F8 is to exclude possible sources west of Brest.

Because of the difference between the forward real world and the backward modelled world it is necessary to loosen the constraints of the local optimisation. This may be done in two steps in such a way that the practical results finally display a great coherence with the ideal results.

Firstly the model, especially with six hourly meteorological input data, cannot reproduce with a great accuracy the 3-hourly behaviour of the real cloud, except in the very special case of F8 not seeing the cloud at all. Then, instead of considering in the simplex algorithm the complete sequence of measurements at F20 or D10, it seems more reasonable to select a few ones to capture the passing of the cloud. We noticed that the ideal results were not significantly different when using complete or partial sequences so that the present strategy may be in fact considered a removal of redundant information. In the case of the Freiburg episode with 8-hourly measurements this removal of redundant information was not necessary.

The strategy, as shown by Fig. 4a, b, d, e enables to obtain practical results very coherent with the ideal ones when F20 and D10 are considered separately. If the complete sequence of measurements at F20 was handled then, as shown in Fig. 4a1, only sources close enough to the station would be compatible. The threshold $Q^{\min }=307 \mathrm{~kg}$ diagnosed for Monterfil would not be coherent with the ideal threshold $Q^{\min }=160 \mathrm{~kg}$. The selection of a few measurements is not so important for D10 probably because the evolution of the signal there has been smoothed on a larger distance to the source. No position at all is jointly compatible with both the sequences of real measurements at F20 and D10, either complete or partial. We still have to loosen the constraints.

Secondly the simplex algorithm is a very constraining tool that must be handled with care when the model is uncertain. 

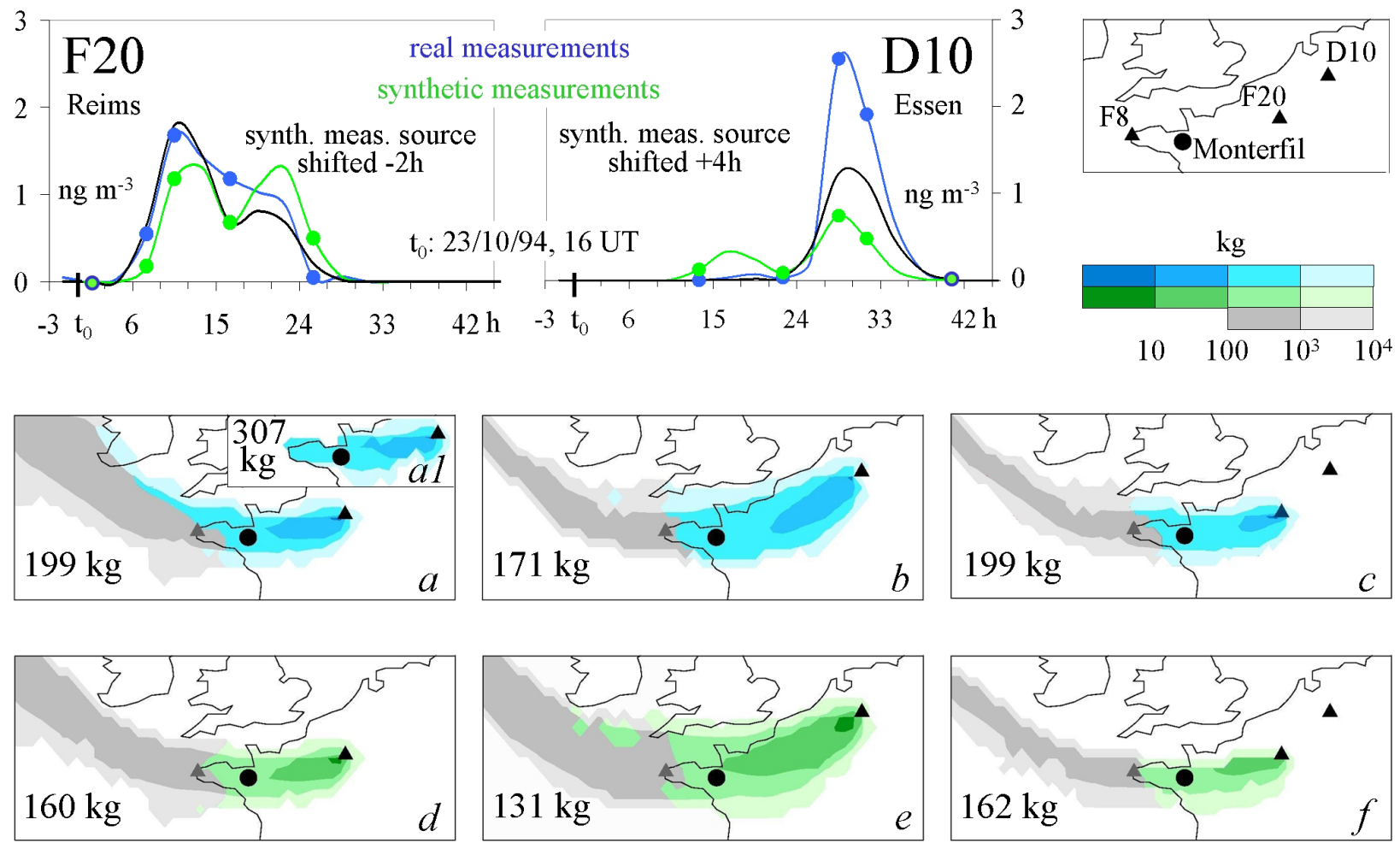

Fig. 4. The upper part of the figure describes the time evolution of the three hourly averaged measurements by stations F20 and D10 after the ETEX-1 release, $340 \mathrm{~kg}$ of pmch from Monterfil during 12 hours beginning on 23 October 1994, 16:00 UT : the real measurements (blue curves) and the synthetic measurements simulated by POLAIR (green curves). A better fit is obtained for the synthetic measurements if the twelve-hour release at Monterfil begins at 14:00 UT for F20, at 20:00 UT for D10 (black curves). The coloured dots indicate the measurements selected as sufficient and not excessive constraints for the investigation of possible point sources at ground or sea level. The lower part of the figure shows the result of the simplex algorithm for the stations represented by a triangle with the selected real, on (a) and (b), or synthetic measurements on (d), (e) and (f). When taken into account, the series of zero-valued measurements obtained at F8 just has the effect of excluding otherwise possible sources indicated by grey colours. (a1) is obtained with the simplex algorithm applied to the complete sequence of real measurements by F20. (c) is obtained as a maximin combination $Q^{\operatorname{maxmin}}$ of (a) and (b); the blue part of (c) is a maximin combination of the blue parts of (a) and (b). The numbers in kg indicate the minimum amount of tracer diagnosed for a source in Monterfil to be compared to $340 \mathrm{~kg}$.

In the model the travel time of the cloud between two stations is calculated with a shift due to slight errors in the speed of the analysed winds. In the case of ETEX1, a shift more than three hours would break the coherence of real measurements with respect to the calculations so that no simulated source would be compatible with them. It seems this is what happened to F20 and D10; as regards F8, a shift would have no consequence. To see that we shifted in fact the twelvehour source in Monterfil in order to obtain a better fit of the real and simulated time evolution of the measurements. As shown in the upper part of Fig. 4, the best fit at F20 is obtained for a source shifted -2 hours, but for D10 the source should be shifted +4 hours.

A possible strategy is to use the simplex algorithm separately for F20 and D10 in order to obtain threshold functions $Q_{\mathrm{F} 20}^{\min }$ and $Q_{\mathrm{D} 10}^{\min }$ and to evaluate a joint threshold constraint as $Q_{\mathrm{F} 20, \mathrm{D} 10}^{\operatorname{maxmin}}=\max \left(Q_{\mathrm{F} 20}^{\min }, Q_{\mathrm{D} 10}^{\min }\right)$. Indeed, if $Q$ is the to- tal amount of tracer released by a point source at position $\boldsymbol{x}$, then necessarily:

$$
\begin{gathered}
Q \geq Q_{\mathrm{F} 20}^{\min }(\boldsymbol{x}) \text { and } Q \geq Q_{\mathrm{D} 10}^{\min }(\boldsymbol{x}) \\
\Longrightarrow Q \geq Q_{\mathrm{F} 20, \mathrm{D} 10}^{\operatorname{maxmin}}(\boldsymbol{x})
\end{gathered}
$$

We called 'maximin' this strategy which is less constraining than the joint management of all measurements from F20 and D10 in the simplex algorithm. Nevertheless, as visible from Fig. 4c, obtained as a maximin of real measurements, and Fig. 4f obtained as a simplex of the analogous synthetic measurements, the practical diagnostics is very close to the ideal one. Note also that the $199 \mathrm{~kg}$ practically diagnosed for Monterfil are consistent with the $340 \mathrm{~kg}$ of the real source.

The maximin strategy is not the only possible one. It is just a very simple example but the idea of joint compatibility of a source with respect to the observations at several places is 
lost. More sophisticated strategies could be proposed to capture such compatibility or incompatibility. In the case of the Freiburg episode, because of the longer sampling intervals, eight hours, we think that no special precaution would be necessary to use the simplex algorithm with several stations, if any. We stress nevertheless that building such figures as Fig. 3 should be considered a pivotal and prudent element of any diagnostics.

Hence, provided some reasonable adaptations are made, the methods described in this paper are robust enough to be handled in an operational context.

\section{Conclusions}

Among the forty CTBT noble gas stations many are settled in industrial areas close to such civilian sources of ${ }^{133} \mathrm{Xe}$ as nuclear plants or hospitals. On the one hand, it is to be expected that sometimes several stations close to each other will simultaneously detect abnormal concentrations corresponding to independent local events. On the other hand, nuclear tests are highly unlikely but would most often be seen by several stations as shown by Hourdin and Issartel (2000) for weak explosions of 1 kiloton. The above method enables to discriminate between both circumstances. A set of positive data produced by independent local events will hardly be compatible with any single point source. For instance, no position is compatible any longer if two zero valued artificial measurements are added in Stockholm just before and just after the positive artificial one thus displaying the rapid evolution tied to a local contamination. In an operational situation, after determining that a set of measurements corresponds to several sources, it is still possible to investigate various selections of stations in order to determine which ones could have seen a test and which other ones were polluted independently.

This investigation would usefully complement the observation of nuclide ratios that are different for nuclear tests and civilian releases. The discriminating ability of the method proposed here is a real asset with respect to the diplomatic and political aims of the CTBT.

It may be tempting to interpret a set of measurements in terms of an optimal position of the source minimising some quadratic cost function. Besides its increasing complexity if a duration of the source is addressed the method would have two important drawbacks. Firstly, as explained in this paper following the suggestions of Penenko and Baklanov (2001), several positions are often acceptable for the source, the problem is not to define a best one but to determine what is possible. Secondly the best position may fail to be good because a best position is still rashly defined in situations of measurements tied to several independent sources. The reader should wonder which of these is the simplest and most natural question when considering a set of observations: 1 ) which sources are compatible? 2) which source minimises a quadratic distance to be defined with the observations?
The methods proposed in this paper pertain to a now flourishing domain of inverse problems with many new ideas (Ternisien et al., 2000). Many studies are currently published about a number of atmospheric species (van Aardenne et al., 2001; Sportisse and Quélo, 2002). These methods generally aim at rebuilding a complex source $\sigma(x, t)$ by means of concentration measurements. The information contained in such measurements $\mu_{k}$ may be summarised into the following equations by means of the associated adjoint retroplumes $\hat{\chi}_{k}^{*}$ :

$\mu_{k}=\int \rho(\boldsymbol{x}, t) \hat{\chi}_{k}^{*}(\boldsymbol{x}, t) \sigma(\boldsymbol{x}, t) d \boldsymbol{x} d t$

This equation or system of equations is linear with respect to the source $\sigma$ and its inversion as such has been proposed by Seibert (2001) in the frame of the CTBT. The system 19 is drastically under-determined. Considering again that the measurement process defines a scalar product we see that measuring the $\mu_{k}$ amounts to determine the orthogonal projection of the function $\sigma(\boldsymbol{x}, t)$ over the retroplumes $\hat{\chi}_{k}^{*}(\boldsymbol{x}, t)$. The real source $\sigma$ cannot be determined exactly but the available information enables to propose some linear combination of the $\hat{\chi}_{k}^{*}$ as an estimation for it. A general theory of such inverse problems, especially the regularisation of the estimation by a 'truncated singular value decomposition' (TSVD), has been addressed by Bertero et al. $(1985,1988)$ in a context dominated by image deblurring purposes.

This is not the approach that has been followed in this paper. We did not try to determine a source $\sigma$, we endeavoured to determine the position $\boldsymbol{x}$ of a point source. The system 19 is linear with respect to $\sigma(\boldsymbol{x}, t)$, not with respect to $\boldsymbol{x}$. In order to explore which positions $\boldsymbol{x}$ were possible positions for a point source, we have degraded the complete linear system 19 into local systems:

$\mu_{k}=\int \hat{\chi}_{k}^{*}(\boldsymbol{x}, t) \dot{Q}(t) d t$

We used then the linearity of the local systems with respect to a positive local source $\dot{Q}(t)$ to build criteria $Q^{\min }(\boldsymbol{x})$ or $Q_{n}^{\min }(\boldsymbol{x})$ that are clearly non-linear functions of the position.

In the frame of the treaty the calculation of a complex source by linear assimilation techniques would be of interest in order to confirm that a set of positive measurements is due to several local events. An important challenge for this assimilation should be to take into account the non-negative constraint: $\sigma(x, t) \geq 0$. This theoretic aim has been investigated by de Villiers et al. (1999).

Acknowledgements. The authors are very grateful to Bertand Cabrit for many fruitful discussions. Graphics have been prepared with the user friendly and public domain graphical package named GrADS originally developed by Brian Dotty (COLA, support@grads.iges.org) and maintained with the help of Mike Fiorino (LLNL). 


\section{References}

Arthur, R. J., Bowyer, T. W., Hayes, J. C., Heimbigner, T. R., McIntyre, J. I., Miley, H. S. and Panisko, M. E.: Radionuclide measurements for nuclear explosion monitoring, 23rd Seismic Research Review: Worldwide Monitoring of Nuclear Explosions, Jackson Hole, Wyoming, 2-5 October 2001.

Baklanov, A.: Modelling of the atmospheric radionuclide transport: local to regional scale, Numerical Mathematics and Mathematical Modelling, INM RAS, Moscow, volume 2, (special issue dedicated to 75-year jubilee of academician G. I. Marchuk), 244 266, 2000.

Bertero, M., de Mol, C., and Pike, E. R.: Linear inverse problems with discrete data, I: General formulation and singular system analysis, II: Stability and regularisation, Inverse Problems, 1, 301-330, 1985 (part I) and 4, 573-594, 1988 (part II).

Brandt, J., Ebel, A., Elbern, H., Jakobs, H., Memmesheimer, M., Mikkelsen, T., Thykier-Nielsen, S. and Zlatev, Z.: The importance of accurate meteorological input fields and accurate planetary boundary layer parameterizations, tested against ETEX1, in ETEX symposium on long-range atmospheric transport, model verification and emergency response, 13-16 May 1997, Vienna, Austria, Nodop editor, Proceedings, European Commission, EUR 17346 EN, 195-198, 1997.

Carrigan, C. R., Heinle, R. A., Hudson, G. B., Nitao, J. J., and Zucca, J. J.: Trace gas emissions on geological faults as indicators of underground nuclear testing, Nature, 382, 528-531, 1996.

CTBTO/PTS: Evaluation of the atmospheric transport modelling tools used at the Provisional Technical Secretariat, Report to the PTS of an Ad-hoc Expert Group on the evaluation of atmospheric models used at the PTS for radionuclide transport analyses, Preparatory Commission for the Comprehensive Nuclear Test Ban Treaty Organization, Vienna, report CTBT/EVA/EG1/1, 73 pages, 2001.

Dantzig, G. B.: Linear Programming and Extensions, Princeton University Press, 1963.

De Geer, L.-E.: Sniffing out clandestine tests, Nature, 382, 528531, 1996

de Villiers, G. nD., McNally, B., and Pike, E. R.: Positive solutions to linear inverse problems, Inverse Problems, 15, 615-635, 1999.

Elbern, H. and Schmidt, H.: A four-dimensional variational chemistry data assimilation scheme for Eulerian chemistry transport modelling, J.G.R. 104(D15), 1999.

Gallardo, L., Olivares, G., Langner, J., and Aarhus, B.: Coastal lows and sulfur air pollution in central Chile, Atmospheric Environment, 36, 3829-3841, 2002.

Goyal, P. and Singh, M. P.: The long term concentration of sulphur dioxide at Taj Mahal due to the Mathura refinery, Atmospheric Environment, 24B, 407-411, 1990.

Hourdin, F. and Issartel, J.-P.: Subsurface nuclear tests monitoring through the CTBT xenon network, Geophysical Research Letters, 27(15), 2245-2248, 2000.

Issartel, J.-P., Cabrit, B., Idelkadi, A., and Hourdin, F.: Localization of sources for events detected during the noble gas equipment test, in Jean and Seibert editors, Informal Workshop on Meteorological Modelling in Support of CTBT Verification, Vienna, 4-6 December 2000.

Joint Research Centre, Etex. The European tracer experiment, European communities, EUR 18143 EN, ISBN 92-828-5007-2, 107 pp., 1998.
Koren, B.: A robust upwind discretization method for advection, diffusion and source terms, in Vreugdenhil and Koren editors, Numerical methods for advection-diffusion problems, Notes on Numerical Fluid Mechanics 45, 117-138, Braunschweig, Vieweg, 1993.

Kunz, C.: Xe-133: ambient air concentrations in upstate New York, Atmospheric Environment, 23, 1827-1833, 1989.

Lewins, J.: Importance, the Adjoint Function, Pergamon Press, 1965.

Louis, J.-F.: A parametric model of vertical eddy fluxes in the atmosphere, Boundary Layer Meteorology, 17, 187-202, 1979.

Marchuk, G. I.: Sopryazhennye Uravneniya i Analiz Slozhnykh Sistem, Moskva, Nauka, 1992, translated in English as: Adjoint Equations and Analysis of Complex Systems, Mathematics and its Applications, Hazewinkel (Ed), Kluwer Academic Publisher, 295, 1995

McCourt, F. R. W., Beenakker, J. J. M., Köhler, W. E. and Kuščer, I.: Nonequilibrium Phenomena in Polyatomic Gases, Dilute Gases, International Series of Monographs on Chemistry, 18, 1 ,Clarendon Press, Oxford, 1990.

Olivares, G., Gallardo, L., Langner, J., and Aarhus, B.: Regional dispersion of oxidised sulfur in central Chile, Atmospheric Environment, 36, 3819-3828, 2002.

Ouahsine, A. and Smaoui, H.: Flux-limiter schemes for oceanic tracers: application to the English Channel tidal model, Comput. Methods Appl. Mech. Engrg. 179, 307-325, 1999.

Penenko, V. and Baklanov, A.: Methods of sensitivity theory and inverse modeling for estimation of source term and nuclear risk/vulnerability areas, Lecture Notes in Computer Science (LNCS), 2074, 57-66, Springer, 2001.

Pudykiewicz, J.: Application of adjoint tracer transport equations for evaluating source parameters, Atmospheric Environment, 32(17), 3039-3050, 1998.

Robertson, L. and Persson, C.: Attempts to apply four dimensional data assimilation of radiological data using the adjoint technique, Radiation Protection Dosimetry, Nuclear Technology Publishing, 50, 333-337 1993.

Sartelet, K. N., Boutahar, J., Quélo, D., Coll, I., and Sportisse, B.: Development and validation of a 3D chemistry transport model POLAIR3D, by comparison with data from ESQUIF campaign, Proc. of the 6th GLOREAM workshop: Global and regional atmospheric modelling, Aveiro, Portugal, 4-6 September 2002.

Seibert, P.: Methods for source determination in the context of the CTBT radionuclide monitoring system, in Jean and Seibert editors, Informal Workshop on Meteorological Modelling in Support of CTBT Verification, Vienna, 4-6 December 2000.

Seibert, P.: Inverse modelling with a lagrangian particle dispersion model: application to point releases over limited time intervals, in Gryning and Schiermeier editors, Air Pollution and its Application XIV, NATO, Kluwer Academic/Plenum Publisher, 3813892001.

Sharan, M., McNider, R. T., Gopalakrishnan, S. G., and Singh, M P.: Bhopal gas leak: a numerical simulation of episodic dispersion, Atmospheric Environment, 29, 2051-2059, 1995.

Spee, E. J.: Numerical Methods in Global Transport-Chemistry Models. PhD thesis, Universiteit van Amsterdam, ISBN 9074795-88-9, 1998.

Sportisse, B., Boutahar, J., Debry, E., Quélo, D., and Sartelet, K.: Some tracks in air pollution modeling and simulation, Revista de la Real Academia de Ciencias, Serie A: Matemáticas (RAC- 
SAM), 96(3), 507-528, 2002.

Sportisse, B. and Quélo, D.: Data assimilation and inverse modeling of atmospheric chemistry, to be published in Sharan editor, Special issue of the Journal of the Indian National Science Academy on Advances in Atmospheric and Oceanic Sciences, 2002.

Sweby, P. K.: High resolution schemes using flux limiters for hyperbolic conservation laws, SIAM J. Numer. Anal., 21, 995-1011, 1984.

Ternisien, E., Roussel, G., and Benjelloun, M.: Blind localization by subspace method for a scattering model, Proc. Sysid 2000, 2000.

Uliasz, M. and Pielke, R.: Application of the receptor oriented ap- proach in mesoscale dispersion modeling, in van Dop and Steyn editors, Air Pollution and its Application VIII, NATO, Plenum Publisher, 399-407, 1991.

van Aardenne, J. A., Builtjes, P. J. H., Hordijk, L., Kroeze, C., and Pulles, M. P. J.: Using wind-direction-dependent differences between model calculations and field measurements as indicator for the inaccuracy of emission inventories, Atmospheric Environment, 36, 1195-1204, 2001.

Wendum, D.: Three long-range transport models compared to the ETEX experiment: a performance study, Atmospheric Environment, 32(24), 4297-4305, 1998. 